were recorded to American Thoracic Society (ATS) standards. Blood was taken for total and specific IgE to hard and soft wood.

Results 269 workers participated (table 1). Most were men $(n=261,97 \%)$, with a mean age of 42.4 years (SD 12.6) and 18.9 (12.8) years woodworking. Mean current wood dust exposure was $1.9 \mathrm{mg} / \mathrm{m}^{3}$ (SD 0.9, IQR 1.4). Current asthma symptoms (CAS, defined as wheezing, nocturnal chest tightness, exertional/nocturnal/resting breathlessness, or asthma medication use within the last 12 months) were common, reported by 123 (46\%). Work-related respiratory symptoms were less common, reported by 29 (11\%). Forty one (18\%) people had a $\mathrm{FE}_{\mathrm{NO}} \geq 40 \mathrm{ppb}$. Only one worker had a positive IgE to soft wood. Ten (4\%) had an $\mathrm{FEV}_{1} / \mathrm{FVC}$ less than the lower limit of normal (<LLN). In adjusted regression models, workers in the highest exposure quartile were at lower risk of work-related respiratory symptoms (WRRS) than those in the lowest quartile (OR $0.16,95 \%$ CI 0.03-0.81). Workers in the low exposure group were more likely to have a $\mathrm{FE}_{\mathrm{NO}} \geq 40 \mathrm{ppb} \quad(\mathrm{OR} 3.59$, 95\% CI 1.09-11.77). However, there was no clear exposure response relationship when looking at percent predicted $\mathrm{FEV}_{1}$ or FVC (for $\mathrm{FEV}_{1} \beta=0.05$, $\mathrm{p}=0.41)$.

Conclusion CAS are common among British woodworkers, reported by nearly half. One fifth fulfilled BTS criteria for high $\mathrm{FE}_{\mathrm{NO}}$ despite low sensitisation rates. The highest exposed were at lower risk for WRRS, suggesting a healthy worker effect. No clear relationship between exposure and lung function was identified. Mechanisms for asthma among woodworkers may not be IgE mediated, and longitudinal studies are needed to clarify the exposure response relationship.

Abstract S105 Table 1 Table showing population characteristics of 269 British woodworkers across exposure quartiles

\begin{tabular}{|c|c|c|c|c|c|}
\hline & $\begin{array}{l}\text { Total } \\
(n=269)\end{array}$ & $\begin{array}{l}\text { Lowest } \\
\text { exposure } \\
(0-1.19 \mathrm{mg} / \\
\left.\mathrm{m}^{3}\right) \mathrm{n}=67\end{array}$ & $\begin{array}{l}\text { Low } \\
\text { exposure } \\
(1.20-2.00 \\
\left.\mathrm{mg} / \mathrm{m}^{3}\right) \\
\mathrm{n}=72\end{array}$ & $\begin{array}{l}\text { Medium } \\
\text { exposure } \\
(2.01-2.32 \\
\left.\mathrm{mg} / \mathrm{m}^{3}\right) \mathrm{n}=63\end{array}$ & $\begin{array}{l}\text { Highest } \\
\text { exposure } \\
(2.33-5.44 \\
\left.\mathrm{mg} / \mathrm{m}^{3}\right) \\
\mathrm{n}=67\end{array}$ \\
\hline \multicolumn{6}{|l|}{ Demographics } \\
\hline $\begin{array}{l}\text { Age, years } \\
\text { (SD) }\end{array}$ & $\begin{array}{l}42.4 \\
(12.6)\end{array}$ & $\begin{array}{l}42.45 \\
(10.51)\end{array}$ & $\begin{array}{l}44.64 \\
(13.69)\end{array}$ & $41.31(13.00)$ & $40.93(12.68)$ \\
\hline Sex, $m(\%)$ & $261(97)$ & 65 (97) & 71 (99) & $62(98)$ & $63(94)$ \\
\hline $\begin{array}{l}\text { Current } \\
\text { smoker (\%) }\end{array}$ & 70 (26) & $17(25)$ & $22(30)$ & $14(22)$ & $17(25)$ \\
\hline $\begin{array}{l}\text { Ever smoked } \\
\text { more than } 1 \\
\text { pack year }(\%)\end{array}$ & $140(52)$ & $34(51)$ & $46(64)$ & $27(43)$ & 33 (49) \\
\hline Exposure & & & & & \\
\hline $\begin{array}{l}\text { Currently uses } \\
\text { RPE (\%) }\end{array}$ & $207(77)$ & $44(66)$ & $55(76)^{*}$ & $51(81)^{*}$ & $57(85)^{*}$ \\
\hline $\begin{array}{l}\text { Mean current } \\
\text { exposure, mg/ } \\
\mathrm{m}^{3} \text { (SD) }\end{array}$ & $1.9(0.9)$ & $\begin{array}{l}0.69(0.37) \\
* *\end{array}$ & $\begin{array}{l}1.74(0.27) \\
* *\end{array}$ & $2.12(0.09)^{* *}$ & $2.98(0.80)^{* *}$ \\
\hline $\begin{array}{l}\text { Total time in } \\
\text { woodworking } \\
\text { industry, years } \\
\text { (SD) } \\
\text { Health }\end{array}$ & $\begin{array}{l}18.9 \\
(12.8)\end{array}$ & $\begin{array}{l}17.95 \\
(11.03)\end{array}$ & $\begin{array}{l}19.71 \\
(12.52)\end{array}$ & $20.86(14.58)$ & $17.11(12.90)$ \\
\hline $\begin{array}{l}\text { Current } \\
\text { asthma } \\
\text { symptoms (\%) }\end{array}$ & $123(46)$ & $34(50)$ & $34(47)$ & $29(46)$ & $26(39)$ \\
\hline
\end{tabular}

\begin{tabular}{|c|c|c|c|c|c|}
\hline $\begin{array}{l}\text { Any work- } \\
\text { related } \\
\text { respiratory } \\
\text { symptom (\%) }\end{array}$ & 29 (11) & 11 (16) & $8(11)$ & 8 (13) & 2 (3) \\
\hline $\begin{array}{l}\text { Work-related } \\
\text { nasal } \\
\text { symptoms (\%) }\end{array}$ & $35(13)$ & $5(8)$ & $8(11)$ & $11(18)$ & $11(16)$ \\
\hline $\begin{array}{l}\text { Work-related } \\
\text { ocular } \\
\text { symptoms (\%) }\end{array}$ & 37 (14) & $6(9)$ & $13(18)$ & $3(5)$ & $15(22)^{*}$ \\
\hline $\begin{array}{l}\text { Physician } \\
\text { diagnosed } \\
\text { asthma (\%) }\end{array}$ & $22(8)$ & $6(9)$ & 10 (14) & $4(6)$ & 2 (3) \\
\hline $\begin{array}{l}\text { Current } \\
\text { asthma under } \\
\text { ECRHS (\%) }\end{array}$ & $40(15)$ & $11(16)$ & $16(22)$ & $8(13)$ & 5 (8) \\
\hline $\begin{array}{l}\mathrm{FEV}_{1} / \mathrm{FVC}<\mathrm{LLN} \\
(\%)\end{array}$ & $10(4)$ & $4(7)$ & $3(5)$ & $1(2)$ & $2(4)$ \\
\hline $\begin{array}{l}\text { Physician } \\
\text { diagnosed } \\
\text { COPD (\%) }\end{array}$ & 4 (2) & $3(5)$ & $0(0)$ & 1 (2) & $0(0)$ \\
\hline $\begin{array}{l}\text { Positive IgE to } \\
\text { hardwood (\%) }\end{array}$ & $0(0)$ & $0(0)$ & $0(0)$ & $0(0)$ & $0(0)$ \\
\hline $\begin{array}{l}\text { Positive IgE to } \\
\text { softwood (\%) }\end{array}$ & 1 (1) & $0(0)$ & $0(0)$ & $1(2)$ & $0(0)$ \\
\hline TlgE, kU (SD) & $\begin{array}{l}133.5 \\
(632.2)\end{array}$ & $\begin{array}{l}138.72 \\
(305.23)\end{array}$ & $\begin{array}{l}95.95 \\
(148.18)\end{array}$ & $\begin{array}{l}236.75 \\
(1223.90)\end{array}$ & $\begin{array}{l}61.40 \\
(108.90)\end{array}$ \\
\hline Atopic $(\%)^{+}$ & $53(20)$ & $13(21)$ & 17 (26) & $16(27)$ & 7 (13) \\
\hline$A M \mathrm{FE}_{\mathrm{no}} \#$ & $\begin{array}{l}27.2 \\
(27.7)\end{array}$ & $\begin{array}{l}24.40 \\
(23.90)\end{array}$ & $\begin{array}{l}32.45 \\
(32.31)\end{array}$ & $24.90(20.29)$ & $26.39(32.45)$ \\
\hline $\begin{array}{l}\text { FENO }>40 \mathrm{ppb} \\
(\%)\end{array}$ & $41(18)$ & 7 (13) & $15(25)$ & $9(17)$ & 10 (16) \\
\hline $\begin{array}{l}\text { Mean FEV } 1 \\
(\%)^{\wedge}\end{array}$ & $\begin{array}{l}99.8 \\
(12.7)\end{array}$ & $\begin{array}{l}99.90 \\
(13.45)\end{array}$ & $\begin{array}{l}98.61 \\
(12.34)\end{array}$ & $99.35(12.25)$ & $\begin{array}{l}101.41 \\
(13.09)\end{array}$ \\
\hline Mean FVC (\%) & $\begin{array}{l}102.9 \\
(12.8)\end{array}$ & $\begin{array}{l}103.48 \\
(12.52)\end{array}$ & $\begin{array}{l}101.16 \\
(12.16)\end{array}$ & $\begin{array}{l}101.63 \\
(12.81)\end{array}$ & $\begin{array}{l}105.41 \\
(13.59)\end{array}$ \\
\hline Mean PEF (\%) & $\begin{array}{l}108.5 \\
(15.9)\end{array}$ & $\begin{array}{l}108.16 \\
(16.91)\end{array}$ & $\begin{array}{l}109.80 \\
(16.63)\end{array}$ & $\begin{array}{l}107.56 \\
(13.86)\end{array}$ & $\begin{array}{l}108.27 \\
(16.14)\end{array}$ \\
\hline $\begin{array}{l}\text { Mean FEV } \\
\text { mls (SD) }\end{array}$ & $\begin{array}{l}3824 \\
(699)\end{array}$ & $3809(658)$ & $3697(720)$ & 3823 (656) & 3986 (739) \\
\hline $\begin{array}{l}\text { Mean FVC, } \\
\text { mls (SD) }\end{array}$ & $\begin{array}{l}4813 \\
(854)\end{array}$ & 4825 (818) & 4658 (899) & $4759(750)$ & 5027 (905) \\
\hline $\begin{array}{l}\text { Mean PEF, mls } \\
\text { (SD) }\end{array}$ & $\begin{array}{l}588 \\
(102)\end{array}$ & 584 (116) & $587(98)$ & 581 (95) & $588(102)$ \\
\hline $\begin{array}{l}{ }^{*} \mathrm{p}<0.05,{ }^{* *} \mathrm{p}< \\
+ \text { +atopy was de } \\
\text { \# Valid } \mathrm{FE}_{\mathrm{No}} \mathrm{m} \\
\wedge \text { Valid spirom }\end{array}$ & $\begin{array}{l}d \text { as } t \\
\text { remen } \\
\text { was I }\end{array}$ & $\begin{array}{l}E>100 \\
\text { e made } \\
\text { ed in } 22\end{array}$ & $\begin{array}{l}\text { workers. } \\
\text { kers. }\end{array}$ & & \\
\hline $\begin{array}{l}\text { D } \\
0 \\
\text { PI } \\
\text { D }\end{array}$ & \multicolumn{5}{|c|}{$\begin{array}{l}\text { DISTRIBUTION OF OCCUPATIONAL AND NON- } \\
\text { OCCUPATIONAL CAUSES IN HYPERSENSITIVITY } \\
\text { PNEUMONITIS DIAGNOSED BY AN INTERSTITIAL LUNG } \\
\text { DISEASE EXPERT PANEL }\end{array}$} \\
\hline
\end{tabular}

1J Mokhlis, ${ }^{1} \mathrm{AS}$ Robertson, ${ }^{2} \mathrm{VC}$ Moore, ${ }^{2} \mathrm{PS}$ Burge, ${ }^{2} \mathrm{GI}$ Walters. ${ }^{1}$ University Hospitals Birmingham NHS Foundation Trust, Birmingham, UK; ${ }^{2}$ Heart of England NHS Foundation Trust, Birmingham, UK

\subsection{6/thoraxjnl-2017-210983.112}

Introduction The aim of this study was to describe the demographic, clinical, exposure history and investigatory Results of hypersensitivity pneumonitis (HP) cases diagnosed by an expert regional NHS interstitial lung disease (ILD) board, to 
determine the extent and distribution of occupational aetiology.

Methods All HP cases identified from a database over a 13 year period were included. The demographic, clinical, exposure history and investigatory Results were reviewed from clinical notes. Cases were categorised in to three groups: occupational, non-occupational and no known cause. Hypothesis testing at the $95 \%$ confidence level was used to identify significant differences between the 3 groups.

Results A total of 127 cases (13 occupational; 34 non-occupational; 80 no known cause) of HP were identified. Men were more likely to experience occupational HP $(p=0.029)$ compared to the other groups. Occupational HP cases were younger $(p=0.002)$, more likely to experience weight loss $(p=0.004)$, to have systemic symptoms $(p=0.007)$ and a recurrence of symptoms $(p<0.001)$. Occupational HP were due to metal working fluids (MWF), birds, mould/fungi, Farmer's lung, and cleaning and treatment sprays. Non-occupational HP were due to birds, mould/fungi in the home and medication. Percentage lymphocyte count in broncho-alveolar lavage (BAL) was significantly raised in occupational HP $(p=0.001)$. Where no causative agent was identified, there was a greater absence of exposure history (13.2\%-32.5\%).

Conclusion Occupations where there is exposure to birds or those working in trades where industrial processes and the use of chemical compounds predominate, are at risk of occupational HP. Birds remain an important cause of non-occupational HP. Clinical features such as weight loss, systemic symptoms and recurrent symptoms should raise a suspicion of an occupational cause of HP. BAL remains an important investigatory tool in HP, especially in occupational HP. An exposure history, especially an occupational history, is mandatory when assessing suspected cases of HP.

\section{S107 SHOULD RESPIRATORY HEALTH BE ASSESSED AS PART OF A PRE-SEASON MEDICAL EVALUATION IN PROFESSIONAL FOOTBALLERS?}

${ }^{1}$ AR Jackson, ${ }^{1} \mathrm{JG}$ Hopker, 'JW Dickinson, ${ }^{2} \mathrm{JH}$ Hull. ${ }^{1}$ University of Kent, Chatham, UK; ${ }^{2}$ Royal Brompton Hospital, London, UK

\subsection{6/thoraxjnl-2017-210983.113}

Introduction and Objectives High rates of exercise induced bronchoconstriction (EIB) are consistently reported in elite endurance athletes, prompting calls to consider screening high risk groups of athletes for respiratory dysfunction. ${ }^{1}$ There are currently no robust data examining the prevalence of respiratory symptoms and airway dysfunction (hyper-reactivity and inflammation) in elite footballers. We therefore undertook this prospective study assessing airway health and the impact of treatment in a cohort of professional footballers undergoing squad pre-season screening.

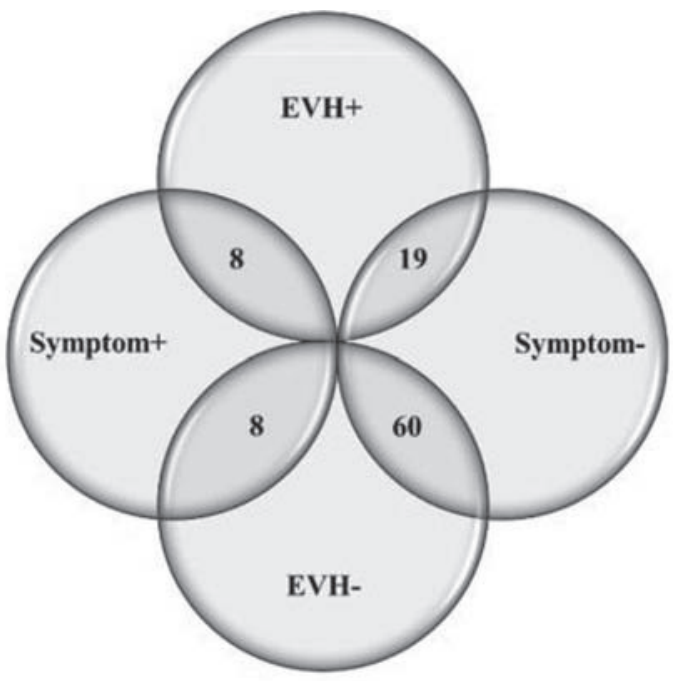

Abstract S107 Figure 1 Relationship between EVH result and presence of respiratory symptoms.

Methods Ninety-seven elite male footballers completed a respiratory assessment including measurement of airway inflammation (FeNO) and screening for EIB using an indirect bronchoprovocation challenge (Eucapnic voluntary hyperpnoea $[\mathrm{EVH}])$. Players demonstrating a positive challenge result $(\mathrm{EVH}+)$ were prescribed appropriate standard asthma therapy directed by EIB severity and underwent repeated assessment after 9 weeks of treatment. Eight players $(\mathrm{EVH}+=3, \mathrm{EVH}-$ $=5)$ also completed a cardiopulmonary exercise test $\left(\mathrm{VO}_{2}\right.$ peak) at the initial and follow-up visits (i.e., post-treatment).

Results Twenty-seven players (29\%) demonstrated EVH+. Of these, ten (37\%) reported no previous history of asthma or EIB. EVH result was not predictable by respiratory symptoms. Seven $(24 \%)$ of the EVH + players attended for follow-up and demonstrated a reduction in FeNO (pre $=85 \pm 61 \mathrm{ppb}$, post $=28$ $\pm 11 \mathrm{ppb}, \mathrm{p}=0.04)$ and a decrease in the fall in $\mathrm{FEV}_{1}$ post EVH (pre $=-22.9 \pm 15.4 \%$, post $=-9.0 \pm 1.6 \%, p=0.018)$. At follow-up, $\mathrm{VO}_{2}$ peak was improved by $3.4 \pm 2.9 \mathrm{ml} . \mathrm{kg}^{-1} \cdot \mathrm{min}^{-1}$ in $\mathrm{EVH}+$ players compared to $0.1 \pm 2.3 \mathrm{ml} \cdot \mathrm{kg}^{-1} \cdot \mathrm{min}^{-1}$ in EVHplayers. Magnitude of inference analysis indicated treatment was possibly beneficial (74\%) for exercise capacity.

Conclusion Elite footballers have a high EIB prevalence, which remains undetected by a symptom based approach to assessment. Treatment with appropriate standard therapy reduces EIB severity, improves airway inflammation and may improve exercise performance. Therefore, the use of objective tests to screen for EIB in this population would be beneficial.

\section{REFERENCE}

1. Dickinson J, Whyte G, McConnell A, et al. Impact of changes in the IOC-MC asthma criteria: A British perspective. Thorax 2005:60:629-632. doi:10.1136/ thx.2004.037499 